Proceedings of the 2012 Winter Simulation Conference

C. Laroque, J. Himmelspach, R. Pasupathy, O. Rose, and A. M. Uhrmacher, eds.

\title{
OPTIMIZATION OF DISTRIBUTED GENERATION PENETRATION BASED ON PARTICLE FILTERING
}

\author{
Nurcin Celik \\ Juan Pablo Sáenz \\ Xiaoran Shi \\ University of Miami \\ 1251 Memorial Drive \\ Coral Gables, FL 33146, USA
}

\begin{abstract}
Distributed generation is small scale power cogeneration within an integrated energy network, that provides system wide and environmental benefits. Network benefits include enhancements to reliability, reduction of peak power requirements, improved power quality and enhanced resilience. Environmental benefits include better land use for transmission and distribution, and reduced ecological impact. Deploying distributed generation affects the power loss in the system and has an associated cost. Therefore, optimization of the penetration level of the distributed generation should consider both goals of minimizing total power loss and minimizing total operational costs. In this study, we propose a novel multi-objective optimization framework based on particle filtering to evaluate the effects of adding distributed generation to a networked system in terms of power loss and operational costs, simultaneously. The proposed framework has been demonstrated on the IEEE-30 bus system yielding to minimal power losses of $2.075 \mathrm{MW}$ and minimal costs of $\$ 547.51$ per hour.
\end{abstract}

\section{INTRODUCTION}

Energy cogeneration and small scale power production, known as distributed generation, is the way in which energy requirements (heating, cooling, lighting, etc.) were met during the original stages of the electric power industry. Technological advances and economies of scale in energy production, transmission and distribution as well as the increasing role of electricity in people's lives, has gradually enabled the development of the current electric network. In the current electric network most of the distributed generation has been replaced by gigawatt scale plants, located away from urban centers and connected through high-voltage transmission and low-voltage distribution lines linking virtually every building in the country. However, some entities (particularly industrial facilities) found it economically beneficial to have their own electric and heating generation systems independently from the central generation units. Furthermore, entities such as hospitals and telecommunication centers, which need highly reliable power, often installed their own generation units as a backup for emergencies. Even though these sources of distributed generation are usually not controlled by the electric utilities the overall electric network may benefit from them, as investments that would have been needed to supply agents with distributed generation may be diverted to fulfill other needs of the network.

Nowadays, technological advances in microturbines, solar panels, reciprocating engines, digital controls and remote monitoring devices (among various others) have increased the opportunities and applications for "next generation" distributed generation, and given customers great flexibility to tailor energy systems to their specific needs. At the same time, electric utility companies are exploring the possibilities that distributed generation may help address some of the requirements of the electric system, promoting greater 


\section{Celik, Sáenz, and Shi}

energy security, economic competitiveness and environmental protection. However, increasing penetration level of the distributed generation may increase security risks and cause crashes in the energy system, such that extreme conditions where there are maximum and minimum loads in the network determine the maximum amount of distributed generation that can be connected given the current network management and technical limitations of the system (Benitez-Rios, Garcia-Lagos, Joya, Atencia, and Sandoval 2011) as well as issues with voltage violations, power losses, power quality, and reliability (Ackermann and Knyazkin 2002). In terms of voltage violations, the presence of distributed generation may help to reduce variations. In terms of power losses, the deployment of distributed generation will generally decrease the amounts of power lost in the system. In terms of power quality, the presence of distributed generation may impact voltage flicker and harmonics. Last but not least, in terms of reliability, the presence of distributed generation may enhance reliability if used to provide backup power or hinder the overall reliability of the grid if it is not properly interfaced with the network. Taking these factors into account, in this study, a novel multi-objective optimization framework based on particle filtering is proposed to evaluate the most beneficial penetration level of distributed generation, minimizing the total operational cost and the total power loss of the system, without posing security risks to the energy network.

The main contributions in this work may be summarized as the introduction of a novel particle filtering framework for multi-objective optimization, and the evaluation of the economic and power loss impacts of the deployment of distributed generation. Multi-objective optimization is required in problems in which optimal decisions are pursued in the presence of trade-offs between two or more conflicting objectives. In these problems, there is seldom a single solution that is optimal for all of the different objectives. Instead, the optimal solution is given by a set of solutions that present different degrees of compromise in each objective, and where improving the value of any single objective implies diminishing the value of at least one other objective. This solution set is known as the Pareto optimal set and is defined as the globally non-dominated set. Multi-objective optimization has been classically addressed through combination or normalization methods that transform the problem to a single-objective optimization problem (Das and Dennis 1998); or through the use of different versions of evolutionary algorithms (Fonseca and Flemming 1995, Zitzler and Thiele 1999, Deb, Pratap, Agarwal, and Meyarivan 2002). The applications of the deployment of distributed generation have been addressed in various works from a single-objective perspective, that include maximizing the levels of distributed generation penetration after solving effects for voltage profiles (Koutroumpezis and Safigianni 2009), the effect on the forecasted future base on different penetration scenarios (Foote, Burns, Elders, and Adult 2005) and the stability and control of the power networks (Benitez-Rios, Garcia-Lagos, Joya, Atencia, and Sandoval 2011), among others. Building on these earlier works the proposed study aims at addressing the deployment of distributed generation from a multi-objective viewpoint.

In this work, a particle filtering framework has been presented for multi-objective optimization by adapting the state space model in two distinct ways. First, state vectors are expanded into matrices where the different dimensions of each objective that is to be optimized are taken into account. Second, in order to increase the accuracy of estimation, we use the non-dominated solution set generated in the sampling stages to update the resampling distributions. This way, as the iterations progress, the algorithm converges to the Pareto front of the sample space. Leveraging this framework, the optimization of distributed generation is evaluated in terms of the total power loss in the system and in terms of the operational costs of such deployment. While the proposed framework has been demonstrated using the IEEE-30 bus test system, it has been constructed in a generic manner so that it can be employed by any networked bus system by inputting its characteristics into the model, and specifying the number of sources of distributed generation that needs to be deployed.

\section{PROPOSED FRAMEWORK}

The power dispatch problem considered in this study involves two distinctive objectives for the implementation of distributed generation. The first objective, defined in Section 2.1, involves the economic load 


\section{Celik, Sáenz, and Shi}

dispatch for the network so that the resources are used in the most cost effective manner. The second objective, detailed in Section 2.2, involves the optimal placement of distributed generation for minimal power loss in the network. Section 2.3 describes the proposed multi-objective optimization algorithm based on particle filtering that was developed in order to fare with the distinct objectives simultaneously. The optimization algorithm leverages the non-dominated solution set generated when sampling from the solution space, in order to improve the accuracy of resampling distributions and obtain better solutions as the iterations progress.

\subsection{Economic Load Dispatch Problem}

The economic load dispatch involves the determination of the output of electric resources to reliably meet the short-term system demand, while minimizing cost and power loss; ensuring that constraints of power balance, and capacity limits in the system are met. The total cost of the generated electricity is provided by ${ }_{i}+{ }_{i} G_{i}+{ }_{i} G_{i}^{2}$, where $i, \quad i$, and ${ }_{i}$, are the cost coefficients of the $i^{\text {th }}$ generator, and $G_{i}$ represents the amount of the real power output obtained from the $i^{\text {th }}$ generator. Here, the generator set includes number of central generation facilities and number of distributed generation units, presented as $G_{i} \in\left\{C G_{1}, C G_{2}, \cdots, C G, D G_{1}, D G_{2}, \cdots, D G\right\}$. The total power loss can be defined as

$$
P_{\text {loss }}={ }_{i=1}^{N}\left(G_{i}-L_{i}\right),
$$

where $P_{\text {loss }}$ represents the total power loss, $N$ is the total number of buses and $L_{i}$ is the real load at bus $i$.

The constraints that must be satisfied in the economic dispatch problem are

$$
\begin{aligned}
& G_{i}^{\min } \leq G_{i} \leq G_{i}^{\max } \quad \forall i, \\
& G_{i}-L_{i}-V_{i}{ }_{j=1}^{N} V_{j}\left[C_{i j} \cos \left({ }_{i}-{ }_{j}\right)+T_{i j} \sin \left({ }_{i}-j\right)\right]=0 \quad \forall i, \\
& Q_{i}-R_{i}-V_{i=1}^{N} V_{j}\left[C_{i j} \sin \left({ }_{i}-{ }_{j}\right)+T_{i j} \cos \left({ }_{i}-{ }_{j}\right)\right]=0 \quad \forall i .
\end{aligned}
$$

The generation capacity constraints are shown in (2), where the real power output of each generator is restricted with minimum $\left(G_{i}^{\min }\right)$ and maximum $\left(G_{i}^{\max }\right)$ capacities. The power balance constraints shown in (3) and (4) ensure that the load provided to the system covers the total demand while considering the energy loss during transmission. Equation (3) addresses the real power balance, where $V_{i}$ is the voltage magnitude at bus $i,{ }_{i}$ is the voltage angle at bus $i, C_{i j}$ is the transfer conductance between buses $i$ and $j$, and $T_{i j}$ denotes the transfer susceptance between buses $i$ and $j$. In (4), which addresses the reactive (imaginary) power balance, $Q_{i}$ is the reactive power generated at the $i^{\text {th }}$ bus, and $R_{i}$ is the reactive load at bus $i$.

The transfer conductance and transfer susceptance are the real and imaginary elements of the bus admittance matrix $Y$. The bus admittance matrix represents the nodal admittance between the different buses of a power system, and is a measure of how easily a current may flow between the buses. The admittance is defined as the inverse of the impedance, which is a measure of the opposition that a circuit presents to the flow of current when a voltage is applied, and extends the concept of electrical resistance to alternating current circuits.

\subsection{Optimal Placement of Distributed Generation}

The location of different sources of distributed generation may be considered optimal if it is such that the amount of power loss in the system is minimized. To this end, we specify the idea how the admittance matrix of the system and the equivalent resistance between slack bus and other buses changed if the distributed 


\section{Celik, Sáenz, and Shi}

power generation unit is added at one of the bus in the system in this section. According to the framework proposed by Wang and Nehrir (2004), in a networked system of $N$ buses, the admittance matrix $Y^{0}$ is defined as

$$
Y^{0}=\left[\begin{array}{ccccc}
Y_{11}^{0} & \cdots & Y_{1 k}^{0} & \cdots & Y_{1 N}^{0} \\
\vdots & & \vdots & & \vdots \\
Y_{N 1}^{0} & \cdots & Y_{N k}^{0} & \cdots & Y_{N N}^{0}
\end{array}\right],
$$

where bus number one is assumed to be the slack bus. Adding distributed generation at bus $j$ causes the admittance to change to $Y$, defined as

$$
Y=\left[\begin{array}{ccccc}
Y_{11}^{0} & \cdots & Y_{1 k}^{0} & \cdots & Y_{1(N-1)}^{0} \\
\vdots & & \vdots & & \vdots \\
Y_{(N-1) 1}^{0} & \cdots & Y_{(N-1) k}^{0} & \cdots & Y_{(N-1)(N-1)}^{0}
\end{array}\right]
$$

where $Y_{11}=Y_{11}^{0}+Y_{j j}^{0}+2 Y_{1 j}^{0}, Y_{1 k}=Y_{1 k}^{0}+Y_{j k}^{0}(k=2, \cdots, j-1), Y_{1 k}=Y_{1(k+1)}^{0}+Y_{j(k+1)}^{0}(k=j, \cdots, N-1)$, $Y_{k 1}=Y_{1 k}(k=2, \cdots, N-1)$.

Assuming that the original load on the system is given by $S_{L}^{0}=\left[S_{L 1}^{0}, S_{L 2}^{0}, \cdots, S_{L N}^{0}\right]$, and the original generated power is given by $S_{G}^{0}=\left[S_{G 1}^{0}, S_{G 2}^{0}, \cdots, S_{G N}^{0}\right]$. Once the distributed generator is added at bus $j$, the new load vector can be presented as $S_{L}=\left[S_{L 1}, S_{L 2}, \cdots, S_{L N}\right]$, where $S_{L i}=L_{i}+j R_{i}, S_{L 1}=0, S_{L i}=S_{L i}^{0}$ for load buses and $S_{L i}=\max L_{i}^{0}-G_{i}, 0$ for P-V buses. In this formulation we assume that at bus 1 , real and reactive power consumed by the load are supplied directly by the generation at that bus whereas the reactive power load at P-V buses may be provided by the external power source at the bus. The power loss in the system after adding distributed generation at bus $j$ is achieved by minimizing $F_{j}={ }_{i=1}^{N} E R_{1 i}(j)\left|S_{L i}\right|^{2}$, where $E R_{1 i}(j)$ is the equivalent resistance between bus $i$ and bus 1 , and is defined as

$$
E R_{1 i}(j)=\left\{\begin{array}{cc}
\operatorname{Real}\left(Z_{11}+Z_{i i}-2 Z_{1 i}\right), & i<j \\
\operatorname{Real}\left(Z_{11}+Z_{(i-1)(i-1)}-2 Z_{1(i-1)}\right), & i>j .
\end{array}\right.
$$

Here $Z$ is the impedance matrix $\left(Z=Y^{-1}\right)$, and it is important to note that $E R_{11}(j)=E R_{1 j}(j)=0$.

In order to add more than one source of distributed generation into the system, the admittance matrix may be updated sequentially so that all of the sources of distributed generation are accounted for. In a similar fashion, the equivalent resistance is to be updated sequentially to account for all of the sources of distributed generation. With this framework there are two ways to ensure that the voltage at each of the buses is held within the acceptable range if the suggested optimal locations lead to the violation of this constraint. The first alternative is to relocate the source of distributed generation from the optimal suggest bus to other buses that are close to the optimal, until the voltage constraints are met. The second alternative to ensure that voltage constraints are not violated, is to decrease the amount of power generated by distributed generation and to optimize the system again.

\subsection{Multi-objective Optimization based on Particle Filtering}

Zhou, $\mathrm{Fu}$, and Marcus (2008) present an optimization framework based on particle filtering that may be extended to multi-objective optimization by adjusting the state of the model from a unique optimal solution to a Pareto optimal solution set. The optimization problem may be represented by

$$
x^{*}=\arg \min f(x)=\arg \min \left(f_{1}(x), f_{2}(x), \cdots, f_{n}(x)\right), \quad x=\left(x_{1}, x_{2}, \cdots, x_{m}\right) \in R^{m},
$$

where $x, m$, and $x^{*}$ are the decision vector, number of decision variables, and Pareto optimal solution set, respectively. 
In this setting, a solution vector is dominated by a solution vector if and only if $\forall i \in 1,2, \cdots, n$ : $f_{i}(\quad) \leq f_{i}(\quad)$ and $\exists j \in 1,2, \cdots, n: f_{j}(\quad)<f_{j}(\quad)$. Furthermore, is said to be covered by if and only if is dominated by or if $f()=f()$ (Zitzler, Deb, and Thiele 2000). A solution vector $x$ is Pareto optimal if it is a non-dominated solution vector.

The state space model that needs to be defined in order to use particle filtering in multi-objective optimization is defined as

$$
\begin{aligned}
x_{k}=x_{k-1}, & k=1,2, \ldots \\
y_{k}=f\left(x_{k}\right)-v_{k} . & k=0,1, \ldots
\end{aligned}
$$

In these equations, $x_{k}=\left(x_{k, 1}, x_{k, 2}, \cdots, x_{k, m}\right)$ is the state of the system at time $k, y_{k}=\left(y_{k, 1}, y_{k, 2}, \cdots, y_{k, n}\right)$ is the measurement taken at time $k, v_{k}=\left(v_{k, 1}, v_{k, 2}, \cdots, v_{k, n}\right)$ is the measurement noise (which is distributed with a pdf $(\cdot))$, and the initial state $x_{0}=x^{*}$.

The Pareto optimal front is generated by an unobserved stationary set of vectors, while it is only possible to observe values on the Pareto optimal front with some noise $\left(y^{*}=f\left(x^{*}\right)\right)$, and values that are covered or dominated by the Pareto optimal front $\left(y_{k} \leq y^{*}\right)$, since $y_{k}=f\left(y_{k}\right)$. For this model,

$$
q_{k}\left(x_{k}\right)=\frac{\left(f\left(x_{k}\right)-y_{k}\right) q_{k-1}\left(x_{k}\right)}{\int\left(f\left(x_{k}\right)-y_{k}\right) q_{k-1}\left(x_{k}\right) d x_{k}}
$$

defines the importance density function.

Here it may be realized that the importance density function is adjusted by the performance of the solutions and generates a new posterior probability distribution as the iterations progress. It is expected that the importance density $q_{k}$ will be getting closer to the density function of $x_{k}$, if $y_{k}$ decreases in relation to $k$. This, seen from an optimization point of view, means that the density defined on the solution space $\left(q_{k}\right)$ becomes more concentrated on the optimal solution as $k$ increases.

Based on the formulation provided in (8) through (11), Figure 1 illustrates the novel particle filtering based optimization framework proposed to solve the multi-objective optimization problem discussed in Sections 2.1 and 2.2. The framework begins by initializing the number of samples for the initial random sampling stage and for the resampling stages, defining the non-dominated set as an empty set, and defining the number of iterations to perform. Once initialization is completed; the data for buses, lines, and cost is read for the performance of the random sampling stage. The admittance matrix is then updated to reflect the distributed generation levels from the random dispatch, and used to calculate the resulting loads and the equivalent resistance. Once the admittance has been updated and the equivalent reactance has been calculated, the resultant power generation as well as the loss is evaluated at the swing bus to ensure the power balance constraints are met.

Once power balance is ensured for all of the samples, the non-dominated solution set is calculated and resampling densities are generated. Three resampling densities are generated where the first one is generated within the samples of the non-dominated solution set, and the other two are generated from the extreme points of the non-dominated solution set and their closest extreme points of the sampling space. The next step is to perform resampling from each distribution. The new samples are then used to update the admittance matrix as the process iterates. Once the desired number of iterations is reached, the final non-dominated solution set is calculated.

\section{EXPERIMENTS AND RESULTS}

In order to establish the validity of the distributed generation penetration optimization framework proposed in this study, we use the IEEE-30 bus test system as an assessment case where the cost data for the generation capacities and parameters of both the central generation and distributed generation units are obtained from (Phonrattanasak 2010). Synchronous generators (rotating energy conversion machines) from microturbine technology are considered as the sources of distributed generation as it provides a reliable 


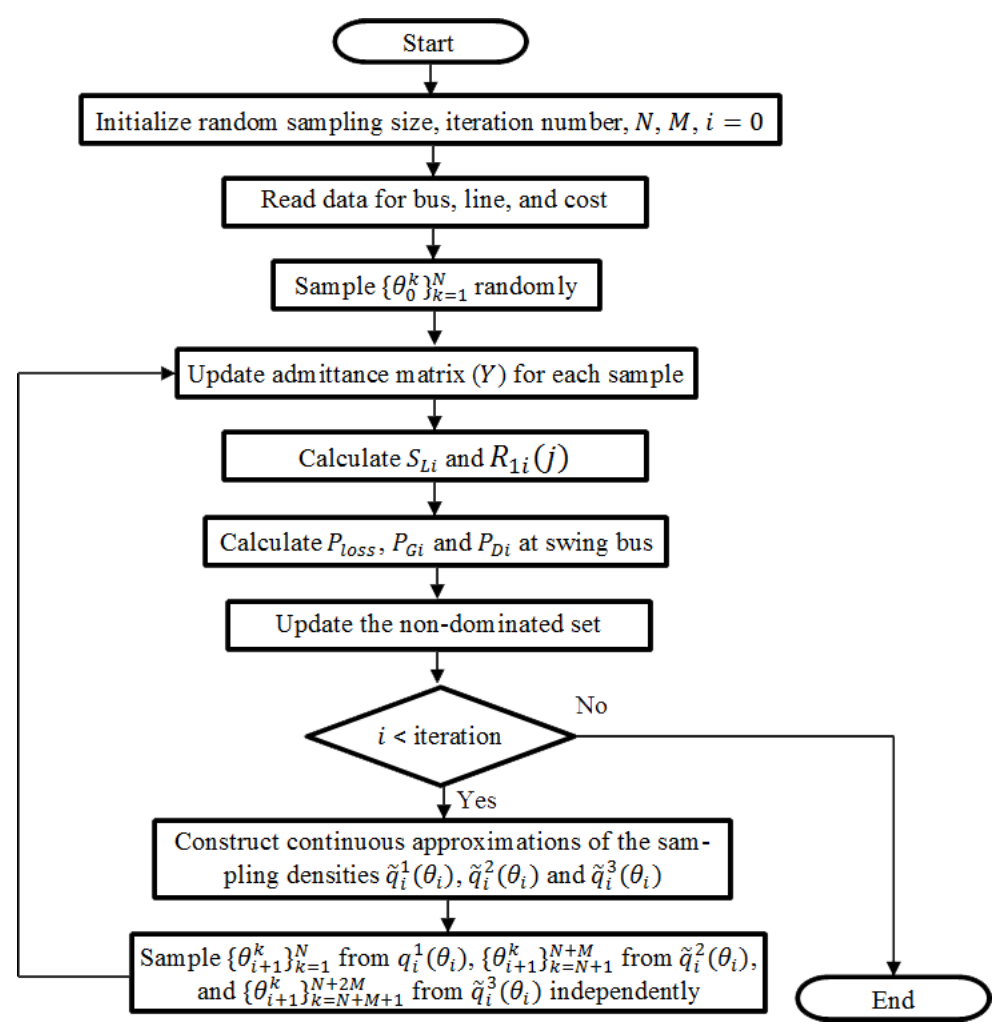

Figure 1: Flowchart of operations performed at proposed particle filtering based optimization algorithm

and easily controllable source of distributed generation and does not depend on environmental factors such as solar irradiation, cloud cover, or wind speed as the case in renewable sources of distributed generation. The modeling of different renewable sources of the energy generation within the proposed framework will be addressed as part of the future venues of this work.

\subsection{IEEE-30 Bus Test System}

IEEE-30 bus test system has been used in the literature and in practice as one of the standard test cases for power systems. The data with the characteristics of the IEEE-30 bus test system has been obtained from the Power Systems Test Case Archive of the Department of Electrical Engineering at the University of Washington (of Washington ). The IEEE-30 bus system represents a part of the American Electric Power System, in Midwestern U.S. The system consists of a total of 30 buses and 41 lines, where there are 6 generation buses, 19 load buses, and 5 buses that neither generate nor request electricity, as shown in Figure 2 .

In the load data for the buses used in equations (2), (3) and (4), the real load ranges from 2.4 MW at buses 3 and 29 to $94.2 \mathrm{MW}$ at bus 5, and the reactive load ranges from 1.2 MVAR at bus 2 to 19 MVAR at bus 5, excluding the buses with no real or reactive load. In the impedance data for the 41 lines used in (5)-(7), the resistance ranges from 0 at lines 11, 12, 13, 14,15, 16 and 27 to 0.3202 at line 38, and the reactance ranges from 0.0236 at line 29 to 0.6027 at line 38 .

The cost parameters related to the power generation, for the different central generation units, as well as for the distributed generation are shown in Table 1, where the capacity for central generation units ranges from $10 \mathrm{MW}$ at buses 8 and 13 to $200 \mathrm{MW}$ at bus 1 , while the distributed generation units have a capacity ranging between 0 and $10 \mathrm{MW}$. 


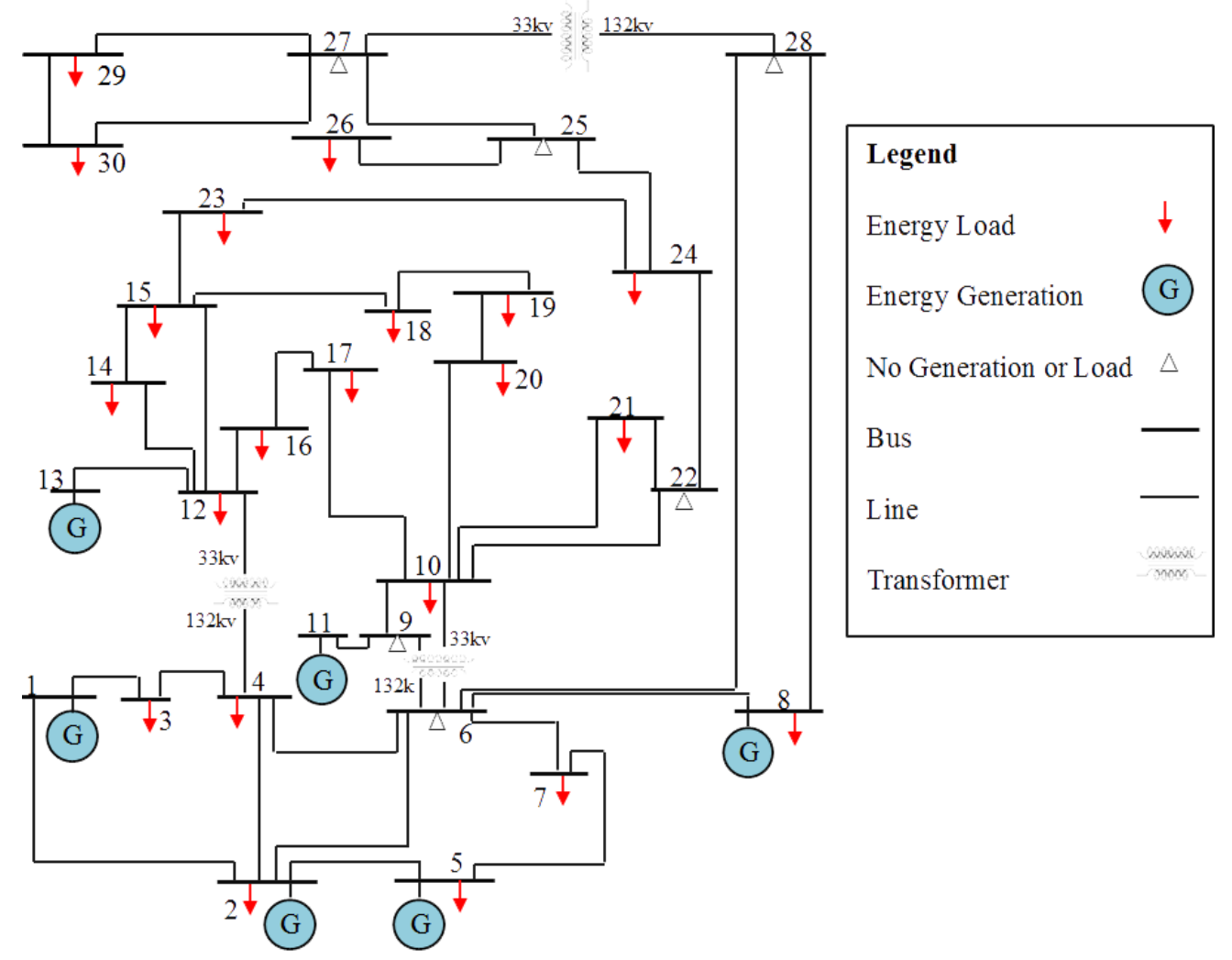

Figure 2: IEEE-30 Bus Test System

\subsection{Optimization of Distributed Generation Penetration}

The particle filtering-based multi-objective optimization framework described in Section 2.3 has been implemented using Matlab R2010b on an Intel Core2 Duo E8600 Computer having 4GB of RAM. In order to provide validation for the proposed framework we have used the case presented by Wang and Nehrir (2004) where a source of 15MW of distributed generation is added to the IEEE-30 system. The results of these simulations are shown in Figure 3, where the minimal power loss is achieved when distributed generation is added at bus 5 with a power loss of $15.482 \mathrm{MW}$, just as in the results presented by Wang and Nehrir (2004) providing validation to the framework's ability to find the best locations to introduce distributed generation to a networked system and minimize the resulting power loss of the electric dispatch.

There are two factors that may affect the performance of the framework, the number of iterations performed and the size of the particle set used for resampling within each iteration. In order to evaluate

Table 1: IEEE-30 cost data.

\begin{tabular}{|c|c|c|c|c|c|c|}
\hline Generation Unit & \multirow{2}{*}{ Bus } & \multicolumn{2}{|c|}{ Capacity } & \multicolumn{3}{c|}{ Cost } \\
\cline { 3 - 7 } & & Min & Max & & & \\
\hline $\mathbf{1}$ & 1 & 50 & 200 & 2 & 2 & 0.00375 \\
$\mathbf{2}$ & 2 & 20 & 80 & 1 & 1.75 & 0.00175 \\
$\mathbf{3}$ & 5 & 15 & 50 & 3 & 1 & 0.00625 \\
$\mathbf{4}$ & 8 & 10 & 35 & 1 & 1.25 & 0.00834 \\
$\mathbf{5}$ & 11 & 12 & 40 & 1.5 & 3 & 0.025 \\
$\mathbf{6}$ & 13 & 10 & 30 & 1 & 3 & 0.025 \\
$\mathbf{D G}$ & - & 0 & 10 & 5 & 1 & 0 \\
\hline
\end{tabular}


Celik, Sáenz, and Shi

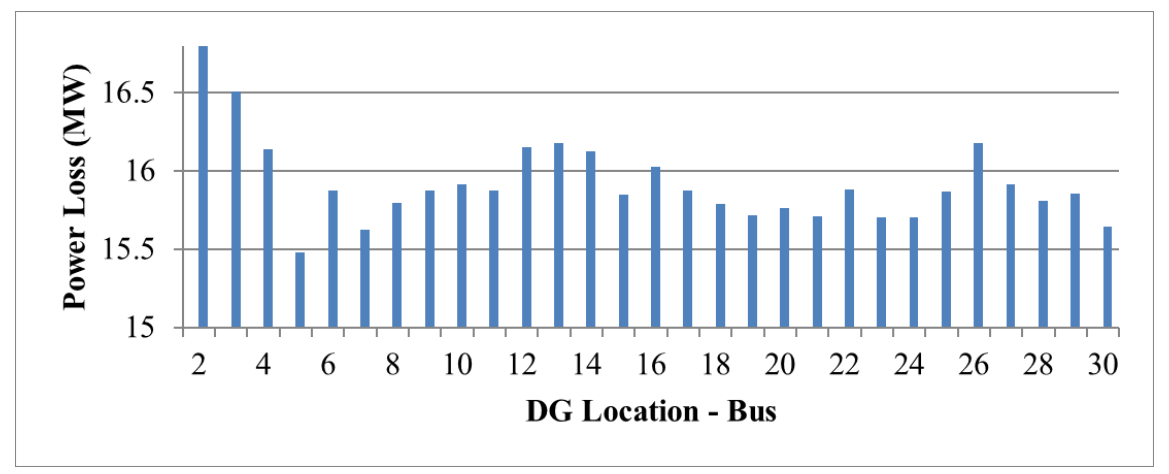

Figure 3: Power loss from placing a 15MW source of distributed generation at different buses within the IEEE-30 system with central generation at buses 1 and 2 .

these two factors, two separate sets of experiments have been performed. The results from these experiments let us to determine appropriate parameters, in terms of number of iterations and particle set sizes, to run the framework in the different proposed scenarios.

To test the effect of the number of iterations on the obtained results, the framework has been evaluated using a fixed initial particle set and a total of 64 particles in the resampling stages. Independent runs with different number of iterations were performed under this setting as shown in Figure 4. The Figure shows that as the number of iterations increases, the solutions with lower cost improve within each non-dominated solution set. Furthermore, there are no significant benefits in increasing the total number of iterations beyond 30, as the different non-dominated solution sets converge to the same solutions.

In order to evaluate the effect of the particle set sizes in the resampling stages, the framework has been evaluated using a fixed initial particle set and 15 iterations. Independent runs with different particle set sizes have been performed under these conditions as shown in Figure 5. In order to evidence the effect of the different particle set sizes clearly, a small number of iterations have been selected. Figure 5 depicts how an increase in the number of particle set sizes impacts (increases) the size of the non-dominated solution set and generates a longer Pareto front with more alternatives. Having a particle set of 32 particles leads to only one non dominated solution with a cost of $\$ 664.76$ per hour and a power loss of $3.467 \mathrm{MW}$, while having a particle set of 192 particles leads to solutions that range from $\$ 665.96$ per hour with a power loss of 3.486 MW to $\$ 604.53$ per hour with a power loss of 4.681 MW.

Based on these results we have conducted experiments using 25 iterations, 100 particles in the initial random sampling stage, 100 particles for the first resampling stage, and 60 particles for the second resampling stage. The experiments have been conducted over five different scenarios where the system is allowed to have "no distributed generation (DG)", "at most one distinct source of DG", "at most two distinct sources of $D G$ ", "at most three distinct sources of $D G$ ", and "a non-predetermined number of sources of DG", respectively. The results associated with each one of these scenarios are summarized below.

\subsubsection{Scenario 1: Predetermined Number of Sources of Distributed Generation}

In this scenario we enable the framework to use a predetermined number of sources of distributed generation, ranging from zero sources of distributed generation, up to three sources of distributed generation. Table 2 shows the minimum cost dispatch from the non-dominated solution sets generated with zero, one, two and three sources of distributed generation.

When the system is not allowed to employ any distributed generation source and rather forced to utilize centralized generation units at all times, the non-dominated solution set achieves the best cost with a resulting power loss is of $5.409 \mathrm{MW}$ at a cost of $596.0181 \$ / \mathrm{h}$. The non-dominated solution set for this number of sources of distributed generation is shown in Figure 6. In this figure, the results presented by (Phonrattanasak 2010) for the economic and environmental dispatch problem are shown in red. In this set 
Celik, Sáenz, and Shi
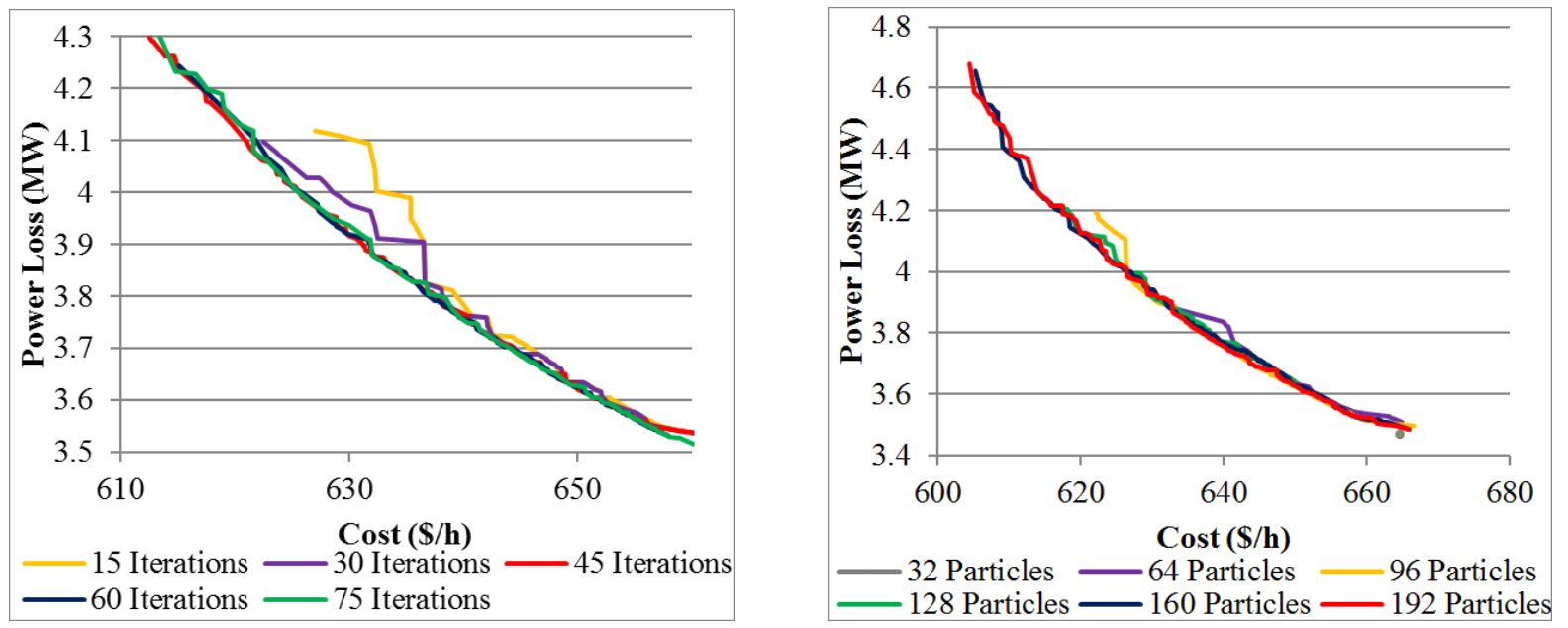

Figure 4: Comparison of different number of itera- Figure 5: Comparison of different particle set sizes. tions.

Table 2: Minimum cost dispatch using a predetermined Table 3: Minimum power loss dispatch using a predenumber of sources of distributed generation.

\begin{tabular}{|c|c|c|c|c|c|}
\hline & \multicolumn{4}{|c|}{ Sources of Distributed Generation } \\
\hline & & 0 & 1 & 2 & 3 \\
\hline \multirow{8}{*}{ 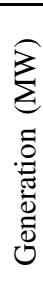 } & Bus 1 & 100.504 & 92.078 & 80.381 & 85.255 \\
\hline & Bus 2 & 80 & 80 & 80 & 80 \\
\hline & Bus 5 & 50 & 50 & 50 & 50 \\
\hline & Bus 7 & 0 & 0 & 7.25 & 6.11 \\
\hline & Bus 8 & 35 & 35 & 35 & 7.35 \\
\hline & Bus 9 & 0 & 9.3 & 10 & 8.72 \\
\hline & Bus 11 & 13.305 & 12 & 12 & 12 \\
\hline & Bus 13 & 10 & 10 & 13.169 & 10.923 \\
\hline \multicolumn{2}{|c|}{ Power Loss (MW) } & 5.409 & 4.978 & 4.4 & 4.608 \\
\hline \multicolumn{2}{|c|}{ Cost $(\$ / h)$} & 596.018 & $\overline{582.642}$ & $\overline{575.976}$ & 553.411 \\
\hline
\end{tabular}
termined number of sources of distributed generation.

of results, the generation at buses $1,2,5,8,11,13$ is $113.919 \mathrm{MW}, 67.425 \mathrm{MW}, 26.671 \mathrm{MW}, 33.843 \mathrm{MW}$, 29.471 MW and 18.836 MW, respectively. The power loss for these generation parameters was calculated to be $5.971 \mathrm{MW}$ at a cost of $667.186 \$ / \mathrm{h}$. It can be seen in the figure that the solution from Phonrattanasak is clearly dominated by the non-dominated set from the proposed framework.

It has been found that the best location for the deployment of one distributed generation unit is at bus 9 , when the system is allowed to employ one source of distributed generation unit, since generation at this bus is part of all of the solutions of the non-dominated set. In this case, the non-dominated solution set achieves the best cost with a resulting power loss of $4.978 \mathrm{MW}$ at a cost of $582.642 \$ / \mathrm{h}$. The non-dominated solution set for one source of distributed generation is depicted in Figure 7.

When the system is allowed to employ two sources of distributed generation units, the best locations for their deployment have found to be buses 7 and 9, as generation at both of these buses is part of all of the solutions of the non-dominated set. Here, the best cost of $575.976 \$ / \mathrm{h}$ is achieved with a resulting power loss of 4.400 MW. Figure 8 shows the non-dominated solution set with two sources of distributed generation.

The results from the proposed framework show that if there is an opportunity to locate three different sources of distributed generation, the optimal results are achieved when locating distributed generation at buses 7 and 9, while not deploying a third source of distributed generation in the network. Figure 9 shows 
Celik, Sáenz, and Shi

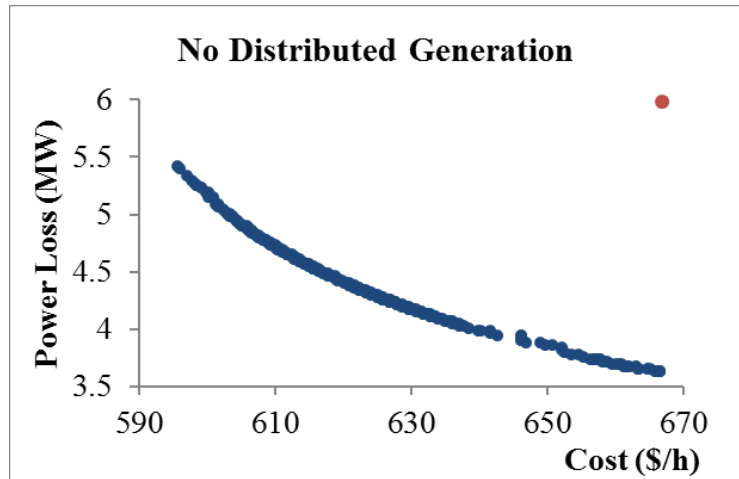

Figure 6: Non-dominated solution set with no sources of distributed generation.

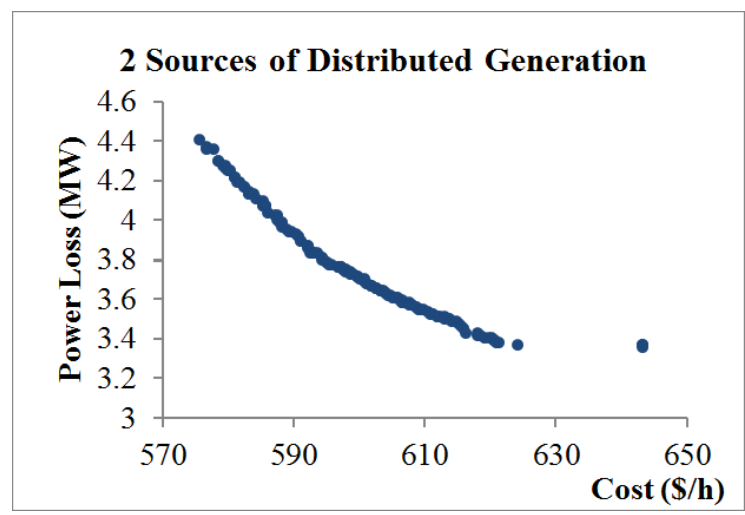

Figure 8: Non-dominated solution set with two sources Figure 9: Non-dominated solution set with three of distributed generation.

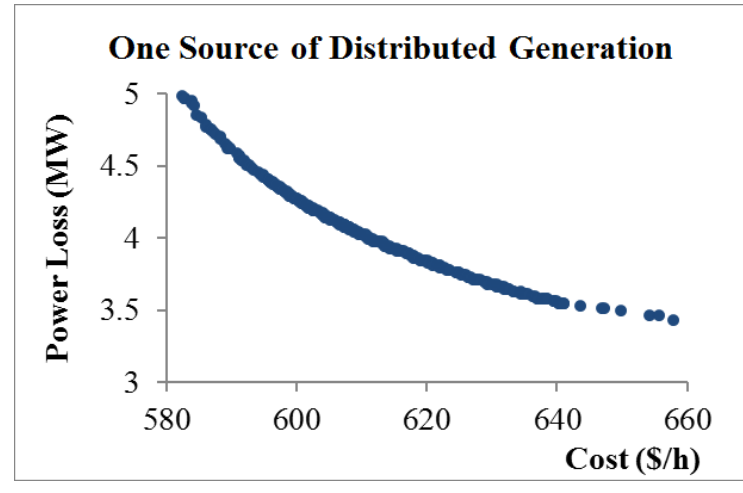

Figure 7: Non-dominated solution set with one source of distributed generation.

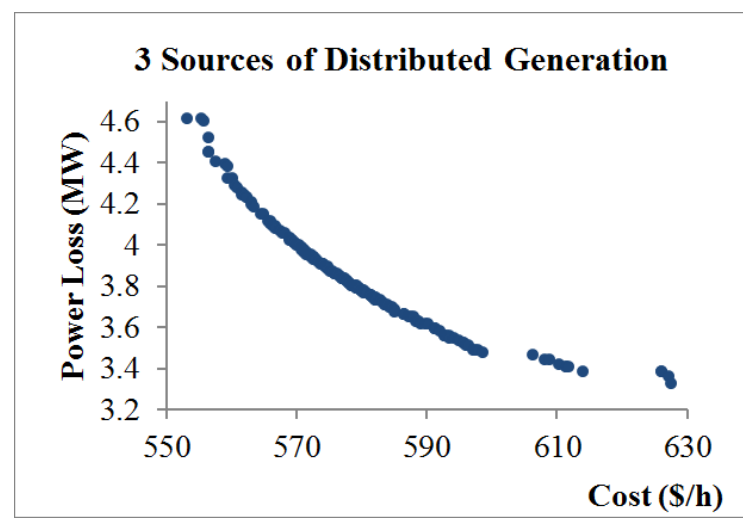

sources of distributed generation.

the non-dominated solution set for three sources of distributed generation units. Here, the best cost of $553.411 \$ / \mathrm{h}$ is reached with a resulting power loss of $4.608 \mathrm{MW}$.

It may be seen that when the goal is to minimize the cost of the energy dispatch the inclusion of one source, two sources, and three sources, of $10 \mathrm{MW}$ of distributed generation may lead to a cost reduction of $2.24 \%, 3.36 \%$ and $7.15 \%$ in the dispatch cost per hour, respectively.

The minimum power loss dispatch, for the different number of sources of distributed generation is shown in Table 3. In this case, when the system is limited to central generation only, the minimum power loss of 3.607 MW is reached at a cost of $666.949 \$ / \mathrm{h}$. When one source of distributed generation is used in the system the minimum power loss dispatch achieves a power loss of $3.42 \mathrm{MW}$ at a cost of $658.182 \$ / \mathrm{h}$. In the case that the system is allowed to use two sources of distributed generation a minimum power loss of 3.35 MW is reached with an associated cost of $643.657 \mathrm{\$} / \mathrm{h}$. Finally, in the case that three sources of distributed generation are used the minimum power loss that can be achieved is of $3.318 \mathrm{MW}$ with a cost of $628.045 \$ / \mathrm{h}$. It may be seen that when the goal is to minimize the power loss of the energy dispatch the inclusion of one source, two sources, and three sources, of $10 \mathrm{MW}$ of distributed generation may lead to a cost reduction of $5.18 \%, 7.13 \%$ and $8.01 \%$ in the dispatch cost per hour, respectively.

\subsection{Scenario 2: Non-predetermined Number of Sources of Distributed Generation Units}

In the case that the system has no limit to the number of sources of distributed generation, the non-dominated solution set achieves the best cost with total central generation of 163.81 MW and total distributed generation 
of $121.99 \mathrm{MW}$. Here, the resulting power loss is $2.400 \mathrm{MW}$ at a cost of $547.514 \$ / \mathrm{h}$. Furthermore, the non-dominated solution set achieves the best power loss with total central generation of $165.665 \mathrm{MW}$ and total distributed generation of $119.81 \mathrm{MW}$, where the resulting power loss is $2.075 \mathrm{MW}$ at a cost of 578.170 $\$ / h$. This scenario's non-dominated solution set is shown in Figure 10.

The comparison of the different levels of distributed generation penetration is provided in Figure 11, where it can be seen that the addition of distributed generation is beneficial in terms of both operational cost and power loss reductions. It is also noted that the non-dominated sets generated with more sources of distributed generation dominate those generated with less distributed generation.

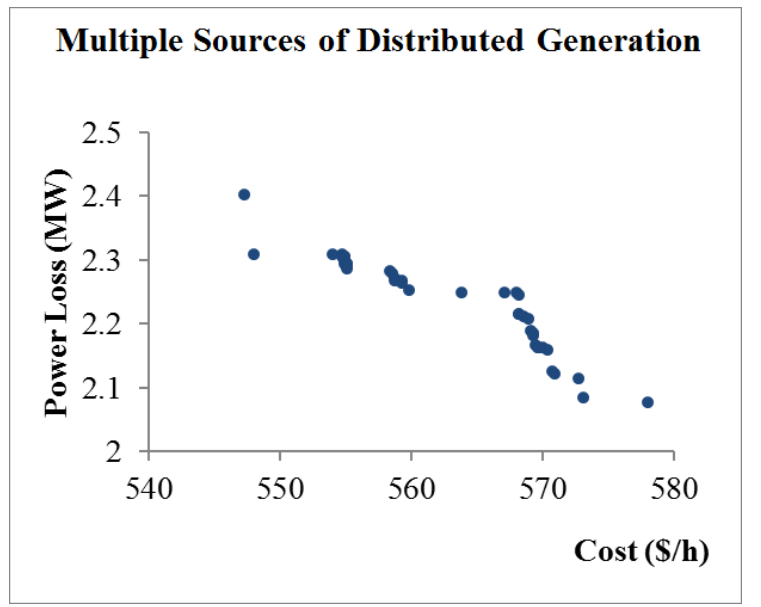

Figure 10: Non-dominated solution set for Scenario Figure 11: Comparison of non-dominated solution 2 (multiple sources of distributed generation).

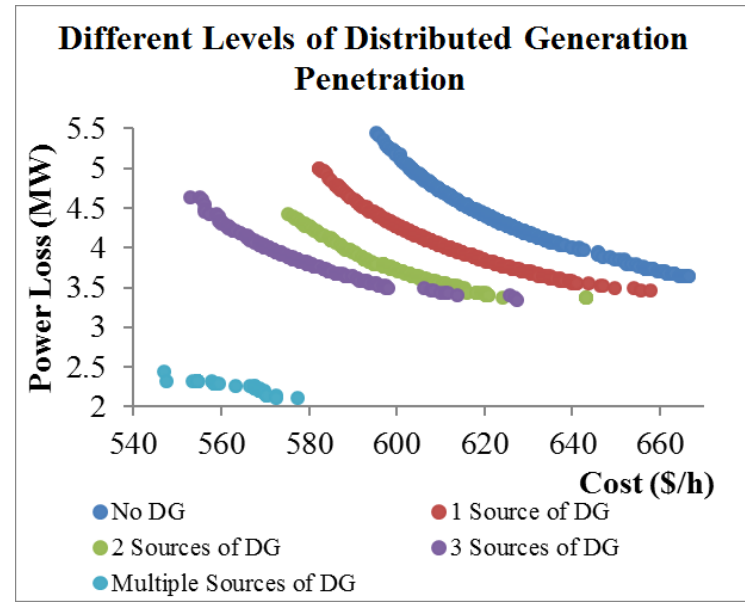

sets with different distributed generation sources.

\section{CONCLUSIONS AND FUTURE WORK}

We have developed a novel comprehensive framework to optimize the penetration level of distributed generation in an energy distribution network based on particle filtering. The proposed framework leverages the information from the generated non-dominated solution sets to enhance the resampling distribution and improve the generated solutions so that as iterations advance, the non-dominated set draws nearer to the Pareto front. The developed framework is able to identify the best locations for any specified number of distributed generation sources in terms of their benefits for power loss reduction and operational costs. The proposed framework has been demonstrated on the IEEE-30 bus system, where it has been found that the best location for the deployment of one source of distributed generation is bus 9, while the best locations for the deployment of two sources of distributed generation are buses 7 and 9. Furthermore, it has been found that given the possibility of using three sources of distributed generation the algorithm's non-dominated solution set is generated using distributed generation only at buses 7 and 9 and the third source of distributed generation is not deployed. The results yield to power losses as low as $2.075 \mathrm{MW}$ and operational costs as low as $547.51 \$ / \mathrm{h}$ when letting the framework use any number of sources of distributed generation. The developed framework has been implemented generically so that it may be implemented on any networked bus system and it may be used to optimize the deployment of any specific number of sources of distributed generation within a network.

Future work for the proposed study includes the incorporation of the environmental implications of deploying different sources of distributed generation into the distributed energy network. Furthermore, the framework may be extended to consider the characteristics of different types of distributed generation, location specifics (i.e., natural resource availabilities such as solar irradiation or wind speed for renewables), and operational policies and restrictions. 


\section{Celik, Sáenz, and Shi}

\section{REFERENCES}

Ackermann, T., and V. Knyazkin. 2002. "Transmission and Distribution Conference and Exhibition 2002: Asia Paci?c". In Transmission and Distribution Conference and Exhibition 2002: Asia Paci?c, Volume 2, $1357-1362$.

Benitez-Rios, F. G., F. Garcia-Lagos, G. Joya, M. Atencia, and F. Sandoval. 2011. "Optimization of distributed generation penetration in distributed power electric systems". In International Conference on Power Engineering, Energy and Electrical Drives, 1-6.

Das, I., and J. E. Dennis. 1998. "Normal-Boundary Intersection: A New Method for Generating the Pareto Surface in Nonlinear Multicriteria Optimization Problems". SIAM Journal on Optimization 8 (3): 631-657.

Deb, K., A. Pratap, S. Agarwal, and T. Meyarivan. 2002. "A fast and elitist multiobjective genetic algorithm: NSGA-II". IEEE Transactions on Evolutionary Computation 6 (2): 182-197.

Fonseca, C. M., and P. J. Flemming. 1995. "An overview of evolutionary algorithms in multiobjective optimization". Evolutionary Computation 3 (1): 1-16.

Foote, C. E. T., G. M. Burns, I. M. Elders, and G. W. Adult. 2005. "Developing distributed generation penetration scenarios". In International Conference on Future Power Systems, 6-18.

Koutroumpezis, G. N., and A. S. Safigianni. 2009. "Optimum distributed generation penetration in a distribution network". In 2009 IEEE Bucharest PowerTech, 1-8.

University of Washington. "Power Systems Test Case Archive UWEE". Accessed Mar. 13, 2012. http: //www.ee.washington.edu/research/pstca/.

Phonrattanasak, P. 2010. "Optimal placement of DG using multiobjective particle swarm optimization". In Proceedings of the 2nd International Conference on Mechanical and Electrical Technology, 342-346.

Wang, C., and M. H. Nehrir. 2004. "Analytical approaches for optimal placement of distributed generation sources in power systems". IEEE Transactions on Power Systems 19:2064-2076.

Zhou, E., M. C. Fu, and S. I. Marcus. 2008. "A Particle Filtering Framework for Randomized Optimization Algorithms". In Proceedings of the 2008 Winter Simulation Conference, edited by S. J. Mason, R. R. Hill, L. Mönch, O. Rose, and J. W. Fowler, 647-654.

Zitzler, E., K. Deb, and L. Thiele. 2000. "Comparison of Multiobjective Evolutionary Algorithms: Empirical Results". Evolutionary Co 8 (2): 173-195.

Zitzler, E., and L. Thiele. 1999. "Multiobjective evolutionary algorithms: a comparative case study and the strength Pareto approach". IEEE Transactions on Ev 3 (4): 257-271.

\section{AUTHOR BIOGRAPHIES}

NURCIN CELIK is an Assistant Professor in the Department of Industrial Engineering at UM. She received her Ph. D. in Systems and Industrial Engineering at University of Arizona. She has received several awards such as the IAMOT Outstanding Research Project Award (2011), UM Provost Award (2011) and the IIE Outstanding Graduate Research Award (2009). Her research interests are in modeling and control of dynamic, complex, and large scale systems and supply chains with a focus on particle filtering methods. She can be reached at celik@miami.edu.

JUAN PABLO SÁENZ is a Ph.D. student in the Department of Industrial Engineering at the University of Miami (UM). He has received the WSC Best Ph. D. Student Paper Award (2011). His research interests are in adaptive modeling and control of distributed electricity power networks. He can be reached at j.saenz4@umiami.edu.

XIAORAN SHI is a Ph.D. student in the Department of Industrial Engineering at UM. Her research interests are in sequential Monte Carlo methods and integrated systems' simulation. She can be reached atx.shi1@umiami.edu. 